\title{
L-Ornithine L-Aspartate (LOLA) for Hepatic Encephalopathy in Cirrhosis: Results of Randomized Controlled Trials and Meta-Analyses
}

\author{
Roger F. Butterworth ${ }^{1}$. Mark J. W. McPhail ${ }^{2}$
}

Published online: 31 January 2019

(c) The Author(s) 2019

\begin{abstract}
This manuscript represents an appraisal of the evidence in support of L-ornithine-L-aspartate (LOLA) for the management and treatment of hepatic encephalopathy (HE) in cirrhosis. Meta-analyses of randomized controlled trials (RCTs) conducted over the last two decades generally reveal evidence of benefit of LOLA in a range of clinical presentations. This included improvement of mental state grade in overt HE (OHE) assessed by West Haven criteria as well as in minimal HE (MHE) assessed by psychometric testing where the oral formulation of LOLA was determined to be particularly effective. However, concerns over study quality were noted in one meta-analysis. Nevertheless, the concomitant lowering of fasting blood ammonia was reported in all RCTs using this endpoint. Network meta-analyses showed that LOLA appears to be comparable (or superior) in efficacy to non-absorbable disaccharides or probiotics. Emerging evidence from single RCTs show efficacy of LOLA for the treatment of post-transjugular intrahepatic portosystemic shunt (TIPSS) HE as well as for secondary HE prophylaxis. These findings provide support for the use of LOLA in the treatment of HE and future trials should focus on the use of LOLA for prophylaxis.
\end{abstract}

\section{Introduction}

Agents with the ability to lower circulating ammonia are the mainstay in the management and treatment of hepatic encephalopathy (HE) in acute liver failure and in cirrhosis. L-Ornithine-L-aspartate (LOLA), a mixture of two endogenous amino acids, is one such agent. Basic research using cultured neural cells, brain preparations from preclinical models of Type C HE as well as patient material, has identified mechanisms responsible for the ammonia-lowering actions of LOLA and these include L-ornithine-induced stimulation of urea synthesis by residual periportal hepatocytes and stimulation of ammonia removal via glutamine synthesis in skeletal muscle.

Mark J. W. McPhail

mark.mcphail@kcl.ac.uk

Roger F. Butterworth

rb@enceph.com

1 Department of Medicine, University of Montreal, 45143 Cabot Trail, Englishtown, NS B0C 1H0, Canada

2 Liver Intensive Therapy Unit, Institute of Liver Sciences, School of Immunology and Microbial Sciences, Kings College London, London, UK
Beneficial effects of LOLA have been reported in over 20 randomized controlled clinical trials (RCTs), the findings of the majority of which have been published in peer-reviewed biomedical journals. The present review focuses on patients with cirrhosis and summarizes the results obtained regarding the efficacy of LOLA as indicated in improvement of mental state and lowering of blood ammonia when compared to placebo or no intervention. Where available, comparison to alternative ammonia-lowering agents such as lactulose, rifaximin, probiotics or branched-chain amino acids (BCAAs) is also made. Data from RCTs, critical reviews and systematic reviews/meta-analyses are included.

\section{Systematic Reviews and Meta-Analyses of Published RCTs}

Results of several systematic reviews and/or meta-analyses on the effects of LOLA in the treatment of HE in cirrhosis have been published in the last two decades. These analyses had a range of objectives and were focused on various subtypes of HE in cirrhosis, oral versus intravenous formulations of LOLA, and differences in experimental design. Extraction of the optimal amount of information and insights regarding the efficacy of LOLA from these analyses 
(including those in abstract form) requires that they be critically reviewed. The first study published in abstract form [1], based upon the findings of five double-blind, placebocontrolled RCTs in 246 patients with cirrhosis (Child-Pugh status A or B) assessed the efficacy of intravenous infusions of LOLA (20-40 mg over 4-8 h for a 7-day period) in terms of improvement of mental state, number connection test-A (NCT-A) scores and hyperammonemia. LOLA treatment resulted in a 3.22-fold greater chance of being free from overt hepatic encephalopathy (OHE) after 7 days of therapy compared to placebo. Treatment also led to significant reduction of post-prandial ammonia during this time.

A second meta-analysis of three randomized trials, judged to be of high quality (using the Jadad score), that included 212 patients with cirrhosis, confirmed the beneficial effect of LOLA in patients with cirrhosis and OHE grades 1 or 2. Higher grades of HE were not included in this study and there was no significant benefit for patients with minimal HE (MHE) [2]. A subsequent systematic review and updated meta-analysis was conducted to assess the efficacy of LOLA in 8 RCTs with 646 patients with cirrhosis (Child-Pugh class $\mathrm{C}$ in $\mathbf{1 5 - 5 0 \%}$ of cases) compared to placebo in six cases, lactulose (1 case) or probiotics (1 case) [3]. In contrast to the results of the previous meta-analysis, LOLA was significantly more effective than placebo and equally effective as lactulose/probiotics for improvement of mental state in both OHE and MHE patients. Significant reductions of fasting ammonia concentrations in LOLA-treated patients were also noted in this analysis.

In a systematic review and meta-analysis of 15 RCTs and 1023 patients published in abstract form, treatment of patients with cirrhosis with LOLA resulted in benefit in the subgroup of patients with acute episodes or chronic HE but not in those with MHE [4].

In view of the inconsistent findings, relatively low patient numbers and/or limited details of procedures due to their publication in abstract form only in many previous analyses, a new systematic review and meta-analysis of 10 RCTs and 919 patients with cirrhosis was designed to assess the efficacy of LOLA for lowering of blood ammonia (Fig. 1) and for improvement of mental state (Figs. 2, 3) [5]. Trial quality and risk of bias was evaluated by means of a novel Jadad/Cochrane paradigm developed by the authors. Subgroup analysis assessed effects on OHE and MHE and, for the first time, the independent efficacy of intravenous versus oral formulations of LOLA was also evaluated. The major findings of this analysis are shown in the form of Forest Plots in Figs. 1, 2 and 3.

Pooled data from the eight trials in which venous ammonia was measured, revealed significant reductions following treatment with LOLA [MD - $17.50(95 \%$ CI - 27.73 to -7.26$)$ ] with either intravenous or oral formulations showing significant effects compared to placebo/no intervention (Fig. 1). Pooled data from the nine trials in which the effects of LOLA treatment on mental state improvement was assessed revealed significant benefit evaluated either by West Haven criteria (WHC) [for OHE, Fig. 2a, b] or by psychometric testing (for MHE, Fig. 3b) in all cases using the Random Effects paradigm for data analysis. Efficacy of the oral formulation of LOLA appeared to be superior to the intravenous formulation in trials of patients with MHE (Fig. 3b). This novel finding was presented at the 2018 EASL meeting in Paris where it was published as an abstract [6].

In an extension of their previous systematic review and meta-analysis, Goh et al. [7] added additional trials for a

Ammonia lowering, all HE trials

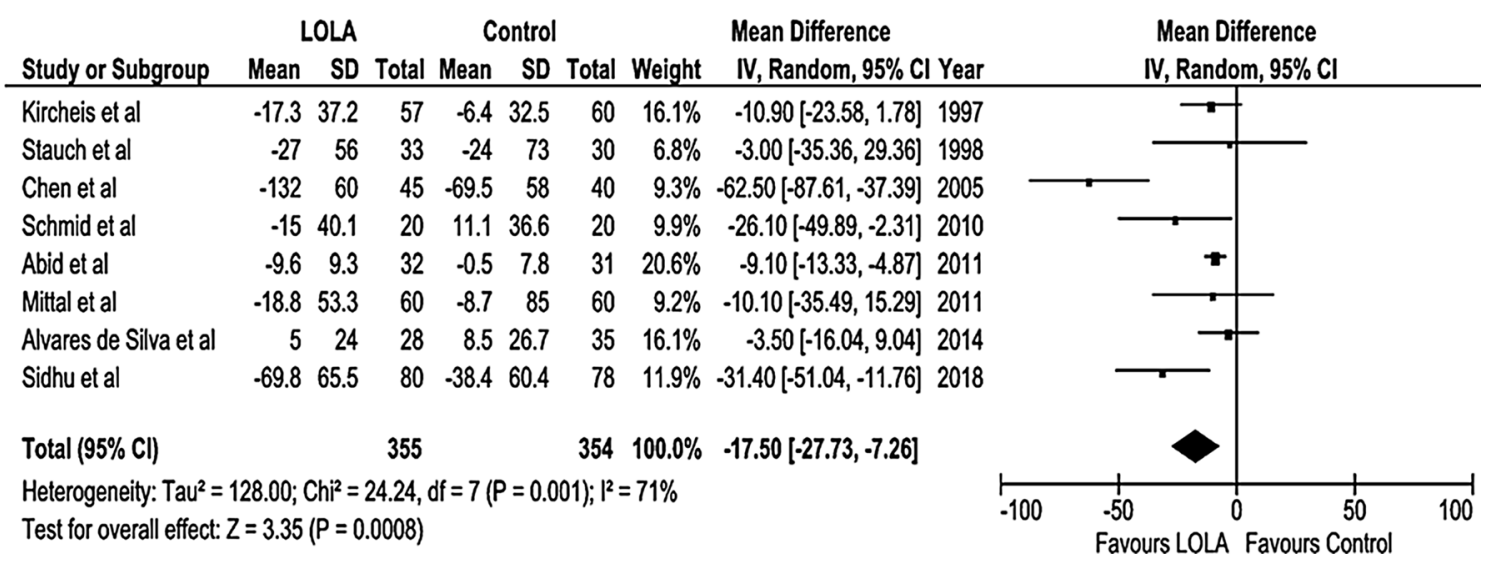

Fig. 1 Forest plot indicating the pooled effect of LOLA versus placebo/no intervention for the lowering of blood ammonia. Trials included: Kircheis et al. [8], Stauch et al. [9], Chen et al. [10], Schmid et al. [11], Abid et al. [12], Mittal et al. [13], Alvares da Silva et al. [14], Sidhu et al. [15], Ahmad et al. [16], Sharma et al. [17] 


\section{Improvement of mental state}

\section{a All HE trials, either formulation}

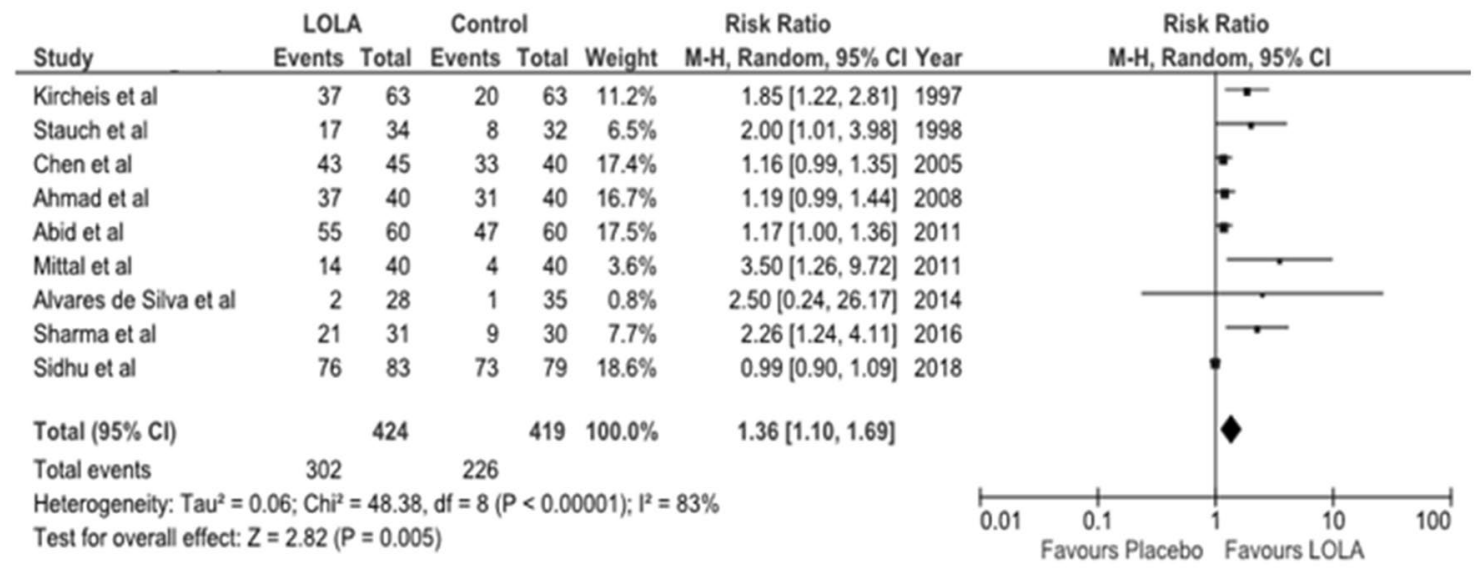

\section{Improvement of mental state b OHE, either formulation}

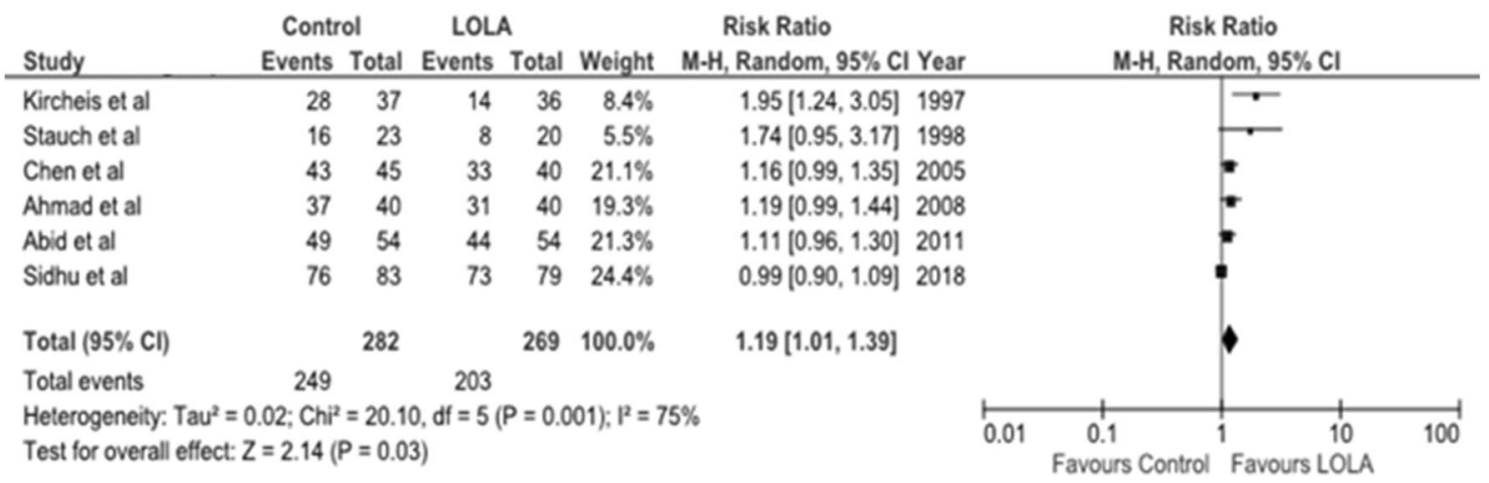

Fig. 2 Forest plots indicating the pooled effect of LOLA versus placebo/no intervention for improvement of mental state in a all HE patients, b patients with OHE. Trials included: Kircheis et al. [8],

total of 36 trials and 2377 patients. Regrettably, most of these additional trials lacked the essential information required for the assessment of treatment outcome and/or risk of bias assessment leading the authors to express uncertainty about the findings. On the other hand, when published or completed trials included in their analysis were assessed, treatment with LOLA was found to be effective for the lowering of blood ammonia [mean dose (MD) - $12.94(-20.04$, $-5.83), Z=3.57, p=00036$ ] confirming the results of the previously published meta-analysis [5]. Comparable confirmatory results were observed for assessments of efficacy of LOLA for the improvement of HE in these completed (RR
Stauch et al. [9], Chen et al. [10], Abid et al. [12], Mittal et al. [13], Alvares da Silva et al. [14], Sidhu et al. [15], Ahmad et al. [16], Sharma et al. [17]

$0.63[0.48,0.83], Z=3.26, p=0.0011)$ or published [RR 0.65 (0.50, 0.85), $Z=3.13, p=0.0017]$ trials.

\section{Comparative Efficacy of LOLA Versus Other Ammonia-Lowering Agents}

Head-to-head trials comparing the efficacy of LOLA with other ammonia-lowering agents have consistently shown that LOLA is equivalent and, in some cases, superior to alternative agents. In an RCT published in 2006, patients randomized to lactulose or LOLA showed comparable 


\section{Improvement of mental state}

\section{a MHE, either formulation}

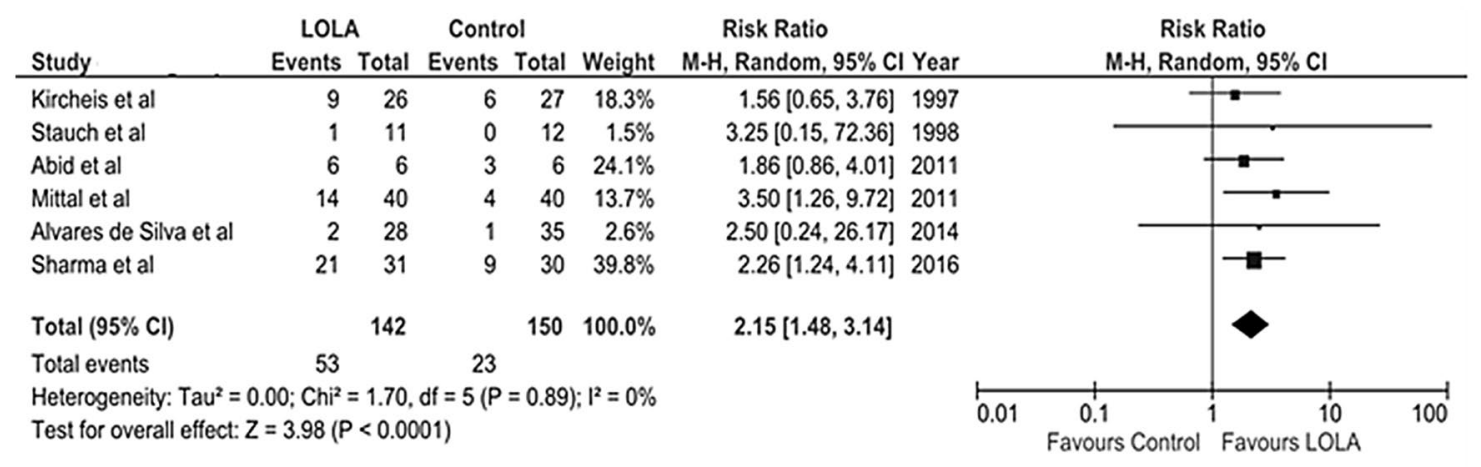

\section{Improvement of mental state b MHE, oral formulation}

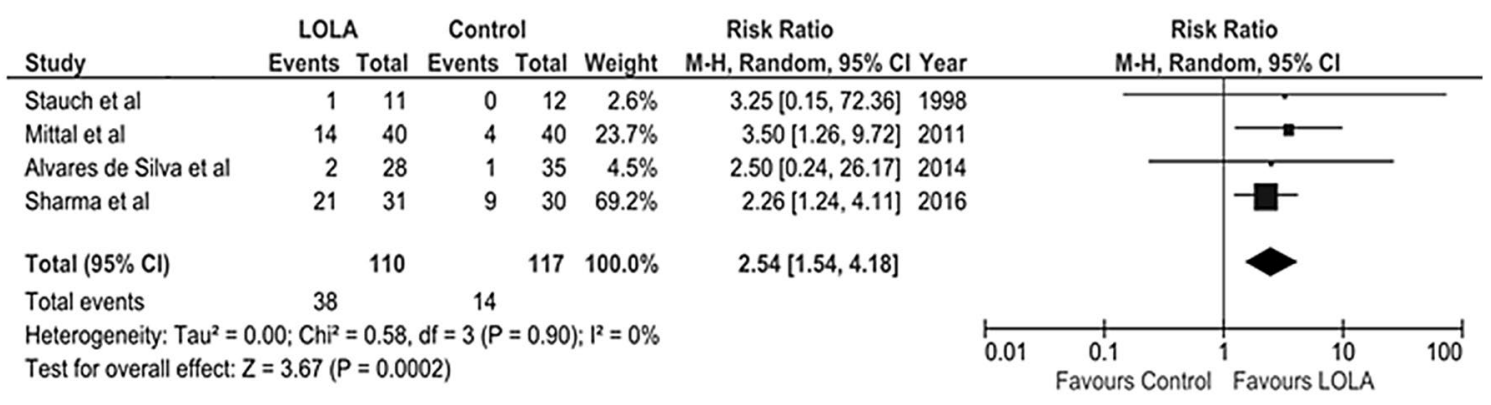

Fig. 3 Forest plots indicating the pooled effect of LOLA versus placebo/no intervention for improvement of mental state in patients with MHE a irrespective of the drug formulation of LOLA $\mathbf{b}$ using the

decreases in serum ammonia levels but only patients in the LOLA treatment group had significant improvements in mental state, number connection test scores, asterixis and EEG activity [18]. Results of subsequent RCTs confirmed the comparable effectiveness of LOLA, lactulose, rifaximin and probiotics for improvement of psychometric test scores and/or critical flicker frequency scores as well as for the prevention of progression to OHE in patients with MHE [13, 14]. These findings were also confirmed in one of the recent meta-analysis on the effectiveness of LOLA [5].

These reports were followed by published accounts of the results of two network meta-analyses comparing the efficacy of LOLA with alternative agents for the treatment of HE in cirrhosis. The effectiveness of various interventions was reported using this approach in 2015 [19] where LOLA, non-absorbable disaccharides, neomycin, rifaximin and BCAAs were compared with respect to clinical efficacy oral formulation. Trials included: Kircheis et al. [8], Stauch et al. [9], Chen et al. [10], Mittal et al. [13], Alvares da Silva et al. [14], Sharma et al. [17]

and ammonia lowering. Only LOLA and BCAA treatments significantly improved clinical symptoms with trends in improvement observed by non-absorbable disaccharides, rifaximin and neomycin compared to observation alone. LOLA showed a statistically significant effect for the lowering of blood ammonia compared to observation alone, but significance was not attained for other comparisons. These findings are summarized in Table 1.

A second network meta-analysis assessed the effectiveness of LOLA, BCAAs, rifaximin, probiotics and lactulose compared to placebo for the treatment of patients with cirrhosis and MHE [20]. In this study, rifaximin, BCAAs and LOLA were superior compared to probiotics and lactulose. Only LOLA and lactulose treatment significantly reduced the risk for progression to OHE in these patients. 
Table 1 Efficacy of LOLA compared to other ammonia-lowering agents by Network meta-analysis

\begin{tabular}{|c|c|c|c|c|c|c|}
\hline Author & Year & No. of trials & No. of patients & Type of HE & $\begin{array}{l}\text { Ammonia- } \\
\text { lowering }\end{array}$ & Comments \\
\hline Zhu et al. [19] & 2015 & 20 & 1.007 & $\mathrm{OHE}$ & NM & $\mathrm{LOLA}=\mathrm{BCAA}>\mathrm{LAC}>\mathrm{NEO}$ \\
\hline Thumburu et al. [20] & 2016 & 27 & 2.056 & MHE & Yes & $\begin{array}{l}\text { RIF }>\text { LAC }>\text { LOLA }>\text { BCAA }^{\text {a }} \\
\text { LAC }>\text { LOLA }>\text { RIF }^{b}\end{array}$ \\
\hline
\end{tabular}

MHE minimal hepatic encephalopathy, $N M$ not measured, $O H E$ overt hepatic encephalopathy

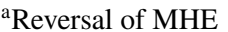

${ }^{b}$ Prevention of OHE

\section{LOLA and HE Prophylaxis Post-TIPSS}

New or worsening HE occurs in 30-50\% of patients following the transjugular intrahepatic portosystemic shunt (TIPSS) procedure [21]. Early post-TIPSS HE is a predictor of poor survival in patients with cirrhosis and complications of portal hypertension and studies conducted so far reveal that neither rifaximin nor lactitol are effective for prophylaxis of HE post-TIPSS [22]. A crossover study in seven patients with previous TIPSS concluded that a single $5 \mathrm{~g}$ dose of LOLA was not effective for alleviation of the acute hyperammonemia resulting from a 20 g glutamine challenge [23]. In contrast, a subsequent RCT of 40 TIPSS patients demonstrated that prophylactic use of intravenous LOLA $30 \mathrm{~g} /$ day for 7 consecutive days was effective in significantly lowering both fasting and postprandial ammonia concentrations [24]. Moreover, psychometric testing using number connection test NCT-A, serial dotting test and line tracing test were all significantly improved in patients in the LOLA treatment group compared to control at days 1, 3 and 7 and patients in the LOLA group tended to have a slower progression to OHE. Previous studies demonstrate substantial increases of liver transaminase enzymes and bilirubin indicative of hepatocellular injury following TIPSS. However, following LOLA treatment the increases in transaminases and bilirubin were significantly attenuated as were increases in MELD scores consistent with the notion that a 7-day prophylactic use of LOLA alleviates post-TIPSS hepatic injury [24]. Novel mechanisms have been proposed to explain these hepato-protective actions of LOLA in patients with cirrhosis [25].

\section{LOLA and HE Prophylaxis in Cirrhosis}

Aside from the apparent effectiveness of LOLA for postTIPSS HE prophylaxis described above, reports of primary or secondary HE prophylaxis by LOLA in patients with cirrhosis have, to date, been limited. A single report of the results of a double-blind RCT of LOLA for secondary HE prophylaxis in 150 patients with cirrhosis has been published [26]. The probability of developing OHE was significantly reduced in the LOLA-treated group and time to first breakthrough of OHE was significantly longer compared to placebo; these benefits were accompanied by improvements in psychometric test scores and critical flicker frequency parameters with significant reductions of arterial ammonia.

\section{LOLA for Treatment of Episodic OHE in Cirrhosis}

In cirrhosis, episodes or "bouts" of OHE are associated with persistent and cumulative deficits of working memory, response inhibition and learning [27]. Moreover, cognitive abnormalities are more prevalent and more severe in patients who have suffered one or more episodes of OHE prior to transplant [28]. Although non-absorbable disaccharides and antibiotics are currently employed, clear evidence for effective management of a single bout of OHE is poor [29]. In a recent RCT of 193 patients having episodic OHE (grades 2-4) with or without precipitating factors, intravenous LOLA $30 \mathrm{~g}$ daily in three divided doses resulted in significant lowering of OHE grade compared to placebo. Mean time taken for recovery from OHE was also significantly less as was venous ammonia and length of hospital stay in LOLA-treated patients [15]. It is important to note that in this trial, all patients, both LOLA-treated and placebo, received lactulose in optimal doses, routes of administration and titration to daily stool production. However, in spite of receiving optimal quantities of lactulose, patients in the placebo arm of the trial remained overtly encephalopathic and hyperammonemic. These lactulose-resistant features were shown to be significantly improved following intravenous LOLA [15].

\section{Summary}

Results of the present review represent an appraisal of the evidence base in support of LOLA for the management and treatment of HE in cirrhosis. All meta-analyses of 
RCTs included in the review showed some level of benefit of LOLA for the treatment of OHE at both low (HE 1, 2) and in high (HE 3,4) grades of severity as assessed by WHC. Efficacy was apparent in a range of clinical presentations that included improvement of mental state grade assessed by WHC in patients with cirrhosis and acute (episodic) or chronic (persistent) forms of HE. Evidence of significant benefit of LOLA was also described in RCTs of patients with MHE assessed by psychometric testing or critical flicker frequency analysis. The oral formulation of LOLA was determined to be particularly effective for the treatment of OHE or MHE. Effective lowering of fasting blood ammonia was reported in all RCTs included in metaanalyses in which it was measured.

Two meta-analyses have expressed concerns over the quality of evidence and therefore confidence in the pooled outcome data [4, 7]. The majority of meta-analyses favor LOLA but certain questions remain. First is the efficacy using modern definitions of HE. Recent expert group guidance has promoted the use of the term "covert HE" for a combined group of previously defined MHE and Grade 1 $\mathrm{HE}$ by WHC grade. The term "overt HE" is reserved for WHC grade 2-4. Most trials and meta-analyses do not use this terminology. Second, where there are concerns and a clinically unanswered question (e.g. pre- and post-TIPSS prophylaxis) large RCTs with sufficient power, funding and quality are required. The use of LOLA for post-TIPSS prophylaxis has been reported in two RCTs the largest of which demonstrated improvements in psychometric test scores, lowering of venous ammonia and, quite unexpectedly, evidence of a direct protective effect of LOLA on TIPSS-related liver injury. An initial report suggests that LOLA may also be effective for non-TIPSS-related secondary prophylaxis in patients with cirrhosis.

Comparison of the results of RCTs examining the efficacy of LOLA for the treatment of OHE compared to other currently available ammonia-lowering agents including BCAAs, lactulose, neomycin, probiotics and rifaximin either by direct head-to-head comparison or by network meta-analysis suggested that LOLA is comparable or superior in efficacy. In patients with MHE, LOLA was found to be comparable to the alternatives for both reversal of deficits in psychometric test scores and for slowing of progression from MHE to OHE.

Taken together, the findings of this review represent a significant basis of support in favor of the use of LOLA for the management and treatment of a wide range of clinical $\mathrm{HE}$ presentations in patients with cirrhosis.

Acknowledgements $\mathrm{MM}$ is grateful to the National Institute for Health Research Biomedical Research Centre at Guy's and St Thomas National Health Service Foundation Trust and King's College London for infrastructure support.

\section{Compliance with Ethical Standards}

Funding This article is published in a special edition journal supplement wholly funded by Merz Pharmaceuticals GmbH, Frankfurt, Germany.

Conflict of interest Dr. Butterworth has performed paid consultancy work on an ad hoc basis for commercial organizations whose products are mentioned in this Supplement including Abbott Laboratories (Switzerland), Umecrine Cognition (Sweden), Vivelix Pharmaceuticals (USA), Merz Pharmaceuticals (Germany), Salix Pharmaceuticals (USA) and Otsuka Pharmaceuticals (Japan). Dr. McPhail has received consultancy fees for statistical analysis from Merz Pharmaceuticals.

Open Access This article is distributed under the terms of the Creative Commons Attribution-NonCommercial 4.0 International License (http://creativecommons.org/licenses/by-nc/4.0/), which permits any noncommercial use, distribution, and reproduction in any medium, provided you give appropriate credit to the original author(s) and the source, provide a link to the Creative Commons license, and indicate if changes were made.

\section{References}

1. Delcker AM, Jalan R, Schumacher M, Comes G. L-Ornithine $\mathrm{L}$-aspartate versus placebo in the treatment of hepatic encephalopathy: a meta-analysis of randomised placebo-controlled trials using individual data. Hepatology. 2000;4:604 (abstract).

2. Jiang Q, Jiang XH, Zheng MH, Chen YP. L-Ornithine L-aspartate in the management of hepatic encephalopathy: a meta-analysis. J Gastroenterol Hepatol. 2009;24:9-14.

3. Bai M, Yang Z, Qi X, Fan D, Han G. L-Ornithine L-aspartate for hepatic encephalopathy in patients with cirrhosis: a metaanalysis of randomized controlled trials. J Gastroenterol Hepatol. 2013;28:783-92.

4. Goh ET, Stokes CS, Vilstrup H, Gluud LL, Morgan MY. L-Ornithine L-aspartate for hepatic encephalopathy: a systematic review with meta-analysis of randomised controlled trials. J Hepatol. 2017;66:131 (abstract).

5. Butterworth RF, Kircheis G, Hilger N, McPhail MJW. Efficacy of L-ornithine L-aspartate for the treatment of hepatic encephalopathy and hyperammonemia in cirrhosis: systematic review and meta-analysis of randomized controlled trials. J Clin Exp Hepatol. 2018;8:301-3. https://doi.org/10.1016/j.jceh.2018.05.004.

6. Butterworth RF, Hilger N, Kircheis G, McPhail MJW. Efficacy of L-ornithine L-aspartate for minimal hepatic encephalopathy in cirrhosis: a systematic review and meta-analysis of randomized controlled trials. J Hepatol. 2018;68(S1):S714. https://doi. org/10.1016/S0168-8278(18)31688-X (abstract).

7. Goh ET, Stokes CS, Sidhu SS, Vilstrup H, Gluud LL, Morgan MY. L-Ornithine L-aspartate for prevention and treatment of hepatic encephalopathy in people with cirrhosis. Cochrane Database Syst Rev. 2018. https://doi.org/10.1002/14651858.cd012410. pub2.

8. Kircheis G, Nilius R, Held C, Berndt H, Buchner M, Goertelmeyer $\mathrm{R}$, et al. Therapeutic efficacy of L-ornithine-L-aspartate infusions in patients with cirrhosis and hepatic encephalopathy: results of a placebo-controlled, double-blind study. Hepatology. 1997;25:1351-60.

9. Stauch S, Kircheis G, Adler G, Beckh K, Ditschuneit H, Goertelmeyer R, et al. Oral L-ornithine-L-aspartate therapy of 
chronic hepatic encephalopathy: results of a placebo-controlled double-blind study. J Hepatol. 1998;28:856-64.

10. Chen M, Li R, Chen C, Gao X. Observation of clinical effect of L-ornithine L-aspartate therapy on liver cirrhosis complicated by hepatic encephalopathy. Chin Libr Class. 2005;2588:06-0718-02.

11. Schmid M, Peck-Radosavljevic M, König F, Mittermaier C, Gangl A, Ferenci P. A double-blind, randomized, placebo-controlled trial of intravenous L-ornithine-L-aspartate on postural control in patients with cirrhosis. Liver Int. 2010;30:574-82.

12. Abid S, Jafri W, Mumtaz K, Islam M, Abbas Z, Shah HA, et al. Efficacy of L-ornithine-L-aspartate as an adjuvant therapy in cirrhotic patients with hepatic encephalopathy. J Coll Phys Surg Pak. 2011;21:666-71.

13. Mittal VV, Sharma BC, Sharma P, Sarin SK. A randomized controlled trial comparing lactulose, probiotics, L-ornithine L-aspartate in treatment of minimal hepatic encephalopathy. Eur J Gastroenterol Hepatol. 2011;23(8):725-32.

14. Alvares da Silva MR, de Araujo A, Vicenzi JR, da Silva GV, Oliveira FB, Schacher F, et al. Oral L-ornithine-L-aspartate in minimal hepatic encephalopathy: a randomized, double-blind, placebo-controlled trial. Hepatol Res. 2014;44:956-63.

15. Sidhu SS, Sharma BC, Goyal O, Kishore H, Kaur N. L-Ornithine $\mathrm{L}$-aspartate in bouts of overt hepatic encephalopathy. Hepatology. 2018;67:700-10.

16. Ahmad I, Khan AA, Alam A, Dilshad A, Butt AK, Shafqat F, et al. L-Ornithine-L-aspartate infusion efficacy in hepatic encephalopathy. J Coll Phys Surg Pak. 2008;18:684-7.

17. Sharma K, Pant S, Misra S, Dwivendi M, Misra A, Narang S, et al. Effect of rifaximin, probiotics and L-ornithine L-aspartate on minimal hepatic encephalopathy: a randomized controlled trial. Saudi J Gastroenterol. 2014;20:225-32.

18. Poo JL, Gongora J, Sanchez-Avila F, Aguilar-Castillo S, GarciaRamos G, Fernandez-Zertuche M, et al. Efficacy of oral L-ornithine L-aspartate in cirrhotic patients with hyperammonemic hepatic encephalopathy. Results of a randomized, lactulose-controlled study. Ann Hepatol. 2006;5:281-8.

19. Zhu GQ, Shi KQ, Huang S, Wang LR, Lin YQ, Huang GQ, et al. Systematic review with network meta-analysis: the comparative effectiveness and safety of interventions in patients with overt hepatic encephalopathy. Aliment Pharmacol Ther. 2015;41:624-35.
20. Thumburu KK, Dhiman RK, Chopra M, Dutta U, Rathi S, Singh M, et al. Comparative effectiveness of different pharmacologicalinterventions for the treatment of minimal hepatic encephalopathy:a systematic review with network meta-analysis. J Clin Exp Hepatol. 2017;7:S6-7. https://doi.org/10.1016/j. jceh.2017.01.010.

21. Roessle M, Haag K, Ochs A, Sellinger M, Noldge G, Perarnau $\mathrm{JM}$, et al. The transjugular intrahepatic portosystemic stent-shunt procedure for variceal bleeding. N Eng J Med. 1994;330:165-71.

22. Riggio O, Masini A, Efrati C, Nicolao F, Angeloni S, Salvatori FM, et al. Pharmacological prophylaxis of hepatic encephalopathy after transjugular intrahepatic portosystemic shunt: a randomized controlled study. J Hepatol. 2005;42:674-9.

23. Rees CJ, Oppong K, Al Mardini H, Hudson M, Record CO. Effect of L-ornithine-L-aspartate on patients with and without TIPS undergoing glutamine challenge: a double blind, placebo controlled trial. Gut. 2000;47:571-4.

24. Bai M, He C, Yin Z, Niu J, Wang Z, Qi X, et al. Randomised clinical trial: L-ornithine-L-aspartate reduces significantly the increase of venous ammonia concentration after TIPSS. Aliment Pharmacol Ther. 2014;40:63-71.

25. Butterworth RF, Gruengreiff K. L-Ornithine L-aspartate (LOLA) for the treatment of hepatic encephalopathy in cirrhosis: evidence for novel hepatoprotective mechanisms. J Liver Clin Res. 2018;5:1044.

26. Varakanahalli S, Sharma BC, Srivastava S, Sachdeva S, Dahale AS. Secondary prophylaxis of hepatic encephalopathy in cirrhosis: a double blind randomized controlled trial of L-ornithine L-aspartate vs placebo. Eur J Gastroenterol Hepatol. 2018;30(8):951-8. https://doi.org/10.1097/meg.0000000000001137.

27. Bajaj JS, Schubert CM, Heuman DM, Wade JB, Gibson DP, Topaz A, et al. Persistence of cognitive impairment after resolution of overt hepatic encephalopathy. Gastroenterology. 2010;138:2332-40.

28. Sotil EU, Gottstein J, Ayala E, Randolph C, Blei AT. Impact of preoperative overt hepatic encephalopathy on neurocognitive function after liver transplantation. Liver Transpl. 2009;15:184-92.

29. Amodio P. Solving doubts about L-ornithine L-aspartate for overt hepatic encephalopathy: whom and how to treat. Hepatology. 2018;67:476-8. 